\title{
Examination of Temperature Influence on Wide-Angle Paratellurite Crystal Acousto-Optic Filters Operation
}

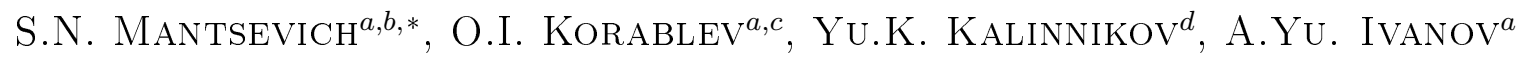 \\ AND A.V. KISELEV ${ }^{a, c}$ \\ ${ }^{a}$ Space Research Institute (IKI), 84/32, Profsoyuznaya, 117997, Moscow, Russia \\ ${ }^{b}$ Faculty of Physics, Moscow State University, GSP-2, Leninskiye Gory, 119992, Moscow, Russia \\ ${ }^{c}$ Moscow Institute of Physics and Technology (MIPT), \\ 9, Institutsky dr., 141700, Dolgoprudny, Moscow Region, Russia \\ ${ }^{d}$ VNIIFTRI, 141570, Mendeleevo, Moscow region, Russia
}

\begin{abstract}
The effect of temperature on a wide-angle paratellurite acousto-optic tunable filter is analyzed on the example of two different acousto-optic tunable filter configurations. The acousto-optic tunable filter operation was between $-50{ }^{\circ} \mathrm{C}$ to $+40{ }^{\circ} \mathrm{C}$; also the survival of an acousto-optic tunable filter device at $-130{ }^{\circ} \mathrm{C}$ was demonstrated. The phase matching ultrasound frequency varies with temperature. We link this temperature shift to photoelastic characteristics of the $\mathrm{TeO}_{2}$. A generalization is made for all wide-angle acousto-optic tunable filters based on TeO 2 crystal. In addition, the temperature influence on the acoustic walk off angle was examined.
\end{abstract}

DOI: $10.12693 /$ APhysPolA.127.43

PACS: 42.25.Fx

\section{Introduction}

Optoelectronic devices based on the acoustooptic (AO) interaction principle are widely used to analyze the optical radiation, in particular the AO tunable filters (AOTF) dedicated to spectral analysis $[1,2]$. The problem of the temperature range, for operation and storage sometimes turns out to be important or even the restrictive factor for AOTF applications. A typical temperature range of $\mathrm{AOTF}$ is $\pm 60^{\circ} \mathrm{C}$. Storage and/or operations below $-80^{\circ} \mathrm{C}$ are of particular importance in space applications [3-5]. Instruments containing the AOTF may be mounted in the places where the temperature stabilization is difficult or even impossible. The effect of temperature on the AOTF operation is far from being fully characterized [6-8], theoretical analysis of influence on the AOTF operation was not done. A consideration is that in $\mathrm{TeO}_{2}$, the phase matching ultrasound frequency depends on the temperature, and the relative shift is $10^{-5}-10^{-4} \mathrm{~K}^{-1}$.

Here we analyze the effect of temperature on the AOTF operation. We tested two wide-angle $\mathrm{TeO}_{2} \mathrm{AOTF}$, developed for deep-space missions. The first one is employed in SPICAM-IR $(1-1.7 \mu \mathrm{m})$, presently in flight onboard Mars Express ESA mission [3]. The second with spectral range $1.15-3.3 \mu \mathrm{m}$ is under development for Lunar Infrared Spectrometer (LIS) and Infrared Spectrometer for ExoMars (ISEM) for the missions Luna 25 and Luna 27, and for ExoMars Rover, respectively.

${ }^{*}$ corresponding author; e-mail: snmantsevich@yahoo.com

\section{Basic relations}

The main characteristic of acousto-optic material is the so-called AO figure of merit $[9,10]$. It is evaluated according to the following equation:

$$
M=\frac{p_{i j}^{2} n_{\mathrm{e}}^{3} n_{\mathrm{o}}^{3}}{\rho V^{3}},
$$

where $\rho$ is the media density, $p_{i j}$ is the matrix of effective photoelastic coefficients, $V$ - acoustic wave velocity along the chosen direction, $n_{\mathrm{e}}, n_{\mathrm{o}}$ - refraction indexes. Some physical properties of the crystal, for example the acoustic wave velocity, depend on the temperature. This dependence takes place as the elastic modules matrix elements, $c_{i j}$ [11-14] change with the temperature. The acoustic wave velocity in crystals is defined by equation:

$$
\left|\Gamma_{j k}-\rho \nu^{2} \delta_{j k}\right|=0
$$

where $v$ - acoustic wave phase velocity, $\delta_{j k}$ - the Kronecker delta, and $\Gamma_{j k}=c_{i j k l} n_{i} n_{l}$ is the second rank tensor. Using data from [11-14], with the help of (2) it is possible to calculate the dependences of acoustic waves velocities on the propagation direction for various temperatures. The propagation direction is set by the azimuthal angle $\theta$, measured from the crystallographic axis $Z$ in (11) $)$ plane or in other words by the cut off angle $\alpha$ measured from [110] axis in the same plane $\alpha=90^{\circ}-\theta$.

In this paper we consider the two cases of the $\mathrm{TeO}_{2}$ crystal cut off angle: $19^{\circ}$ and $12.5^{\circ}$. The dependence $V(\theta)$ varies with the temperature in a complicated way. Also the temperature influences on acoustic walk off angle $\psi$. This effect is presented in Fig. 1 that shows the dependence of $\psi$ shift (the difference between $\psi$ at normal conditions and $\psi$ at low temperatures) on $\theta$ in (11̄0) plane. This shift may be more than $1 \mathrm{deg}$, and may influence the AO diffraction characteristics. 


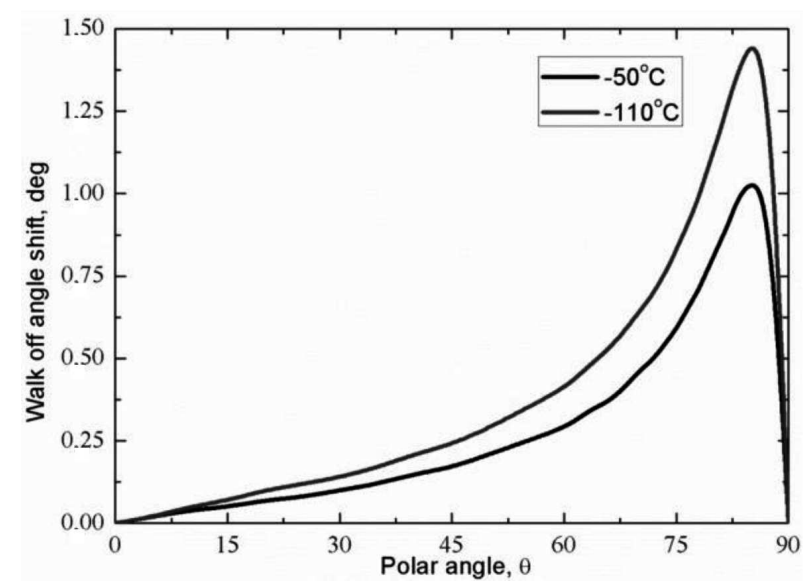

Fig. 1. Dependences of walk off angle for slow acoustic wave velocity on the direction in the (110) crystallographic plane for $-50{ }^{\circ} \mathrm{C}$ (black curve) and $-110^{\circ} \mathrm{C}$ (dashed curve).

\section{AOTF temperature tests}

Formal temperature requirements for Mars Express mission are typical: $-30^{\circ} \mathrm{C} \ldots+40^{\circ} \mathrm{C}$ operational; $-50{ }^{\circ} \mathrm{C} \ldots+60^{\circ} \mathrm{C}$ survival. The thermal tests of the SPICAM AOTF were performed in a climatic chamber. The AOTF transfer function was measured for the wavelengths of $1.13,1.36,1.37$, and $1.53 \mu \mathrm{m}$.

The testing of LIS AO module (hereafter AOM) was done to find out whether it could withstand the temperature requirements for the Lunar and Mars surface missions. The check of the AOM operation consisted of measuring the AOTF transfer function at $1.15 \mu \mathrm{m}$ (79-80 MHz). At each measurement the AOM power consumption was checked in the whole operation range 25-80 MHz (Fig. 2).

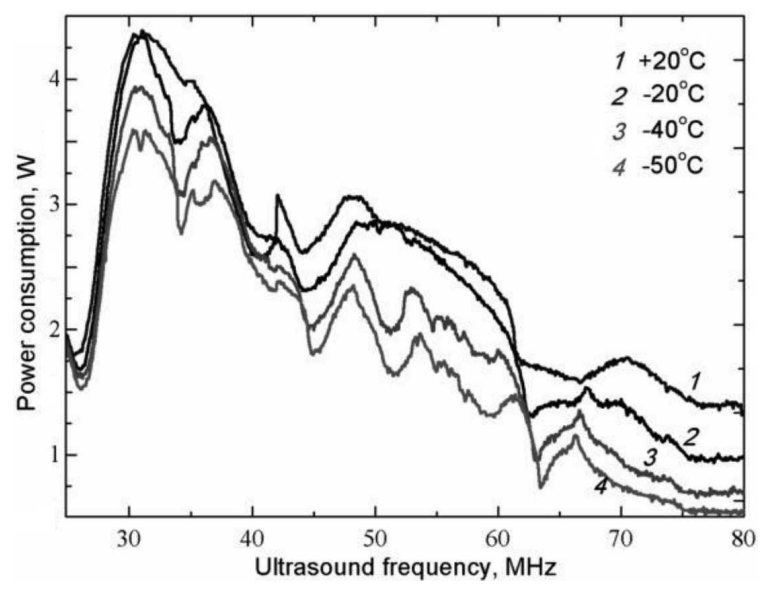

Fig. 2. The LIS AOM power consumption dependences on ultrasound frequency recorded during the thermo-vacuum tests in the range between $+40{ }^{\circ} \mathrm{C}$ and $-50{ }^{\circ} \mathrm{C}$.

We followed the same approach to check the integrity of the LIS/ISEM prototype AOTF in the extended temperature range, to confirm survival temperature of $-130^{\circ} \mathrm{C}$ for the Mars surface mission. Careful inspection of the AOTF crystal did not show any damage or degradation neither in the crystal, nor in the transducers.

\section{Results and discussion}

The phase matching frequency becomes lower with decreasing temperature, in accordance with Eq. (3):

$$
\sin \theta_{\mathrm{B}}=-\frac{\lambda f}{2 n_{\mathrm{i}} V}\left[1+\frac{V^{2}}{\lambda^{2} f^{2}}\left(n_{\mathrm{i}}^{2}-n_{\mathrm{d}}^{2}\right)\right] .
$$

Here $\theta_{\mathrm{B}}$ is the Bragg angle; $n_{\mathrm{i}}$ and $n_{\mathrm{d}}$ are the crystal refraction indices for the incident and diffracted optical waves. We have therefore ignored the temperature dependence of the $\mathrm{TeO}_{2}$ dispersion in the present study.

The overview of temperature influence at AOTFs is presented in Table. In the tested LIS prototype AOTF operation temperature range $\left(-50 \ldots+40^{\circ} \mathrm{C}\right)$ the frequency shift $(\Delta f)$ amounts to $0.42 \mathrm{MHz}$, while the filter pass-band is $0.18 \mathrm{MHz}$. In the case of SPICAM AOTF the cutoff angle is larger, and the dependence of the $V$ on the temperature is smaller. But the phase matching frequency shift is still significant: for the range $-20 \ldots+20^{\circ} \mathrm{C}$ it equals $0.19 \mathrm{MHz}$ that is approximately twice as large as the passband of $0.1 \mathrm{MHz}$.

TABLE

Summary of the test results.

\begin{tabular}{c|c|c}
\hline \hline Instrument & SPICAM & LIS \\
\hline cut off angle $[\mathrm{deg}]$ & $19^{\circ}$ & $12.5^{\circ}$ \\
temp. range $\left[{ }^{\circ} \mathrm{C}\right]$ & $-50 \ldots+40$ & $-50 \ldots+40$ \\
$v$ at normal conditions $[\mathrm{m} / \mathrm{s}]$ & 898.5 & 757.8 \\
temp. variation of $v[\mathrm{~m} / \mathrm{s}]$ & $2.45 \mathrm{~m} / \mathrm{s}$ & $5 \mathrm{~m} / \mathrm{s}$ \\
measured $\Delta f[\mathrm{kHz} / \mathrm{k}]$ & $2.19 \pm 0.03$ & $5.17 \pm 0.12$ \\
calculated $\Delta f[\mathrm{kHz} / \mathrm{K}]$ & $2.91 \pm 0.27$ & $6.1 \pm 0.22$
\end{tabular}

The phase matching frequency was computed for the values of the $c_{i j}$ measured in [11-14]. In Fig. 3a,b we compare the theoretical dependences of the phase matching frequency on temperature computed with the use of Eqs. (2), (3) and experimental elastic coefficients from [13] with those obtained experimentally. In the temperature range considered the phase-matching frequency shift for a wide-aperture AOTF depends linearly on the temperature. The relative shift amounts to $2.5 \times 10^{-5} \mathrm{~K}^{-1}$ for SPICAM and $6.6 \times 10^{-5} \mathrm{~K}^{-1}$ for the LIS prototype. A significant scatter of the theoretical points is caused by the accuracy of the $c_{i j}$ values presented in [13]. Despite the lower accuracy of the theoretical results, the corresponding relative shift of the phase-matching frequency is noticeably larger in both cases, and amounts to $3.2 \times 10^{5}$ and $7.9 \times 10^{5} \mathrm{~K}^{-1}$ for SPICAM and LIS AOTF configurations. This bias of 20 $30 \%$ might be related to the temperature variation of the $\mathrm{TeO}_{2}$ dispersion, ignored in the present analysis.

Besides the effect of the temperature on the AO interaction, the power consumption of the device depends on temperature, too. The AO diffraction efficiency for 

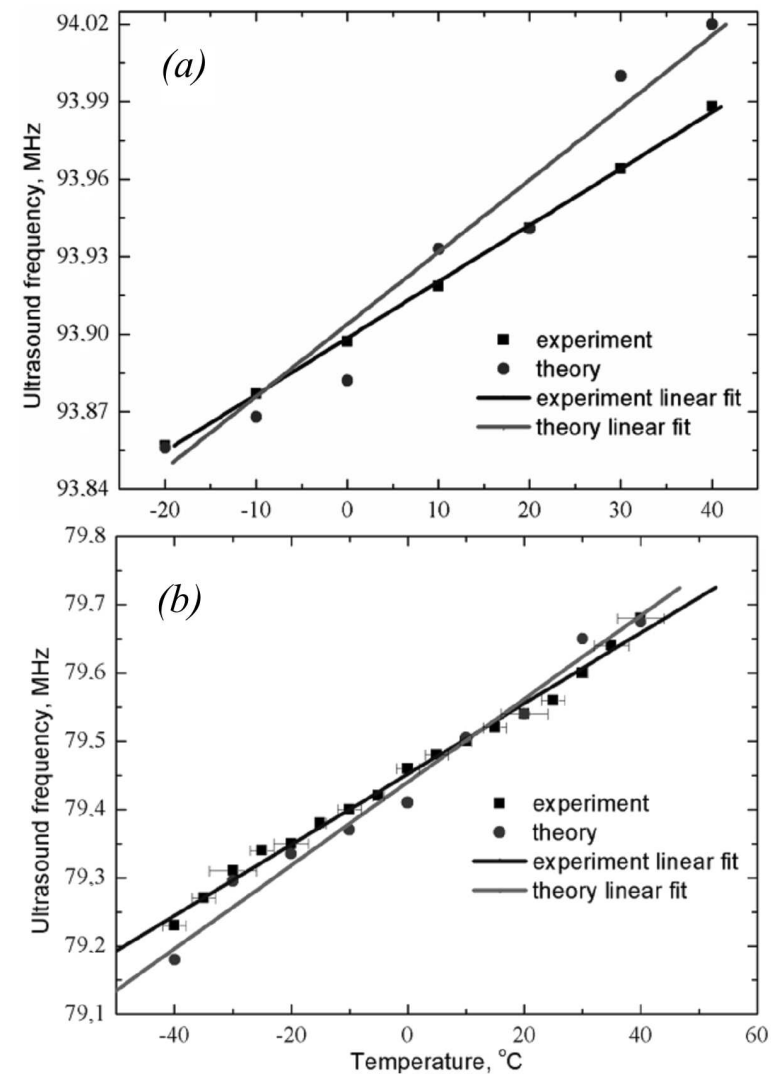

Fig. 3. Phase matching frequency in function of temperature for two AOTF devices. (a) SPICAM-IR AOTF; the optical wavelength of incident light is $1.53 \mu \mathrm{m}$. Calibration results [13] are marked with solid squares. (b) LIS prototype AOTF. The optical wavelength of incident light is $1.15 \mu \mathrm{m}$. Test results are marked with solid squares with error bars.

longer optical wavelengths, corresponding to lower ultrasound frequencies, is smaller than that for shorter optical wavelengths. This defines higher power consumption at low ultrasound frequencies, as in Fig. 2. The AOM power consumption was monitored during the thermal tests in the range between $+20^{\circ} \mathrm{C}$ and $-50^{\circ} \mathrm{C}$. Generally the power consumption decreases with the temperature. The reduction is the most significant at $-40^{\circ} \mathrm{C}$ and $-50^{\circ} \mathrm{C}$. It varies from $10-15 \%$ at longer wavelengths up to $75 \%$ at shorter wavelengths. The decrease of the maximal AO diffraction efficiency at low temperatures following the decrease in the AOM power consumption is also apparent in measured LIS transfer functions. At $-40{ }^{\circ} \mathrm{C}$ the diffraction efficiency is reduced from $\approx 80 \%$ to $\approx 40 \%$, while the power consumption falls to $75 \%$ of the value obtained at normal conditions (Fig. 2).

\section{Conclusions}

We have analyzed the effect of temperature influence on the operation of wide-angle $\mathrm{TeO}_{2}$ AOTFs theoretically and experimentally. We use the results of two spaceborne AOTF devices testing in an extended temperature range. Three effects of the temperature are considered, the shift of the phase matching frequency, the shift of acoustic walk off angle, and the AO efficiency. It is shown that for the two cut-off angles in $\mathrm{TeO}_{2}(1 \overline{1} 0)$ the temperature depression causes a phase matching frequency shift towards lower ultrasound frequencies. This shift depends on the cut-off angle. The phase matching frequency is changing with temperature linearly. The results obtained can be further generalized to all wideangle AOTF's fabricated on the base of $\mathrm{TeO}_{2}$. Comparing the measurements to theoretical estimations we confirm that the shift of the phase matching frequency is mostly explained by the temperature modification of the slow acoustic wave in $\mathrm{TeO}_{2}$. During the tests of the LIS AOTF prototype we achieved extremely low AOTF non-operational temperatures, down to $-130^{\circ} \mathrm{C}$. After tests AOTF appeared fully operational, without loss in optical or electrical properties.

\section{Acknowledgments}

The testing of SPICAM-IR was done by S. Guibert in Service d'Aeronomie. This paper is supported by RSCF grant 14-22-00042.

\section{References}

[1] I.C. Chang, Proc. SPIE 90, 12 (1976).

[2] V.B. Voloshinov, Ultrasonics 31, 333 (1993).

[3] O.I. Korablev, J.-L. Bertaux, A. Fedorova, D. Fonteyn, A. Stepanov, Y. Kalinnikov, A. Kiselev, A. Grigoriev, V. Jegoulev, S. Perrier, E. Dimarellis, J.P. Dubois, A. Reberac, E. Van Ransbeeck, B. Gondet, F. Montmessin, A. Rodin, J. Geophys. Res. E 111, E09S03 (2006).

[4] C. Pilorget, J.-P. Bibring, Planet. Space Sci. 76, 42 (2013).

[5] O.I. Korablev, Yu.K. Kalinnikov, A.Yu. Titov, A.V. Rodin, Yu.V. Smirnov, M.A. Poluarshinov, E.A. Kostrova, A.V. Kalyuzhny A.Yu. Trokhimovskii, I.I. Vinogradov, A.A. Fedorova, A.Yu. Ivanov, A.A. Venkstern, V.V. Barke, O.Z. Rosté, J. Opt. Technol. 78, 317 (2011).

[6] V. Balakshy, V.B. Voloshinov, V.A. Karasev, V.Y. Molchanov, V. Semenkov, Proc. SPIE 2713, 164 (1996).

[7] J. Runhua, Z. Zhenqiao, L. Xiaohua, Z. Shaoqun, H. Zhifeng, Z. Huaichun, Ultrasonics 52, 643 (2012).

[8] P. Maak T. Takacs, A. Barocsi, E. Kollar, P. Richter, Ultrasonics 51, 441 (2011).

[9] Y. Ohmachi, N. Uchida, N. Niizeki, J. Acoust. Soc. Am. 51, 164 (1972).

[10] H. Schweppe, Ultrasonics 8, 84 (1970).

[11] T. Yano, A. Watanabe, J. Appl. Phys. 45, 1243 (1974).

[12] Y. Ohmachi, N. Uchida, J. Appl. Phys. 41, 2307 (1970).

[13] I.M. Silvestrova et al., Phys. Status Solidi 101, 437 (1987).

[14] H. Ledbetter, R.G. Leisure, A. Migliori, J. Betts, H. Ogi, J. Appl. Phys. 96, 6201 (2004). 